\title{
Effect of Mach number on Wall Pressure Flow Field for Area Ratio 2.56
}

\author{
Syed Ashfaq ${ }^{1}$ and S. A. Khan ${ }^{2}$ \\ ${ }^{I}$ (Research Scholar, Department of Mechanical Engineering, JJT University, Rajasthan, India \& \\ Associate Professor, Department of Mechanical Engineering, AAEMF's COE \& MS, Pune, India) \\ ${ }_{2}^{2}$ (Principal, Department of Mechanical Engineering, Bearys Institute of Technology, India)
}

\begin{abstract}
This paper presents the experimental results on the flow characteristics of a suddenly expanded flow from the convergent nozzle for subsonic flow. In the present study micro jets were used to investigate the effect of micro jets on wall pressure flow field in the enlarged duct. Accordingly an active control in the form of four micro jets of $1 \mathrm{~mm}$ orifice diameter located at $90^{\circ}$ intervals along a pitch circle diameter of 1.3 times the nozzle exit diameter in the base region was employed. The Mach numbers of the present studies were $M=0.9$, 0.8 , and 0.6 and the area ratio (ratio of area of suddenly expanded duct to nozzle exit area) studied was 2.56 . The length-to-diameter (i.e. L/D) ratio of the sudden expansion duct was varied from 10 to 1 . From the results, it is seen that the flow in the base region is dominated by the waves, the general perception what we have that correctly expanded flow will be from waves is proved to be wrong; also, it is found that for $L / D$ in the range $L / D$ $=10$ and 8 the flow remains oscillatory mostly for all the Mach numbers. However, these oscillations are suppressed gradually either with the increase in the L/D ratio in the range 3 to 6 or with decrease in the level of inertia level. The present study explicitly reveals that, the wall pressure in a suddenly expanded axi-symmetric duct can be controlled by employing micro jets. Since the flow field in the enlarged duct remained undisturbed hence, we can say that the control in the form of micro jets don't disturb the field.
\end{abstract}

Keywords: Active control, Area ratio, Base pressure, L/D ratio, Micro jets, Static wall pressure

\section{INTRODUCTION}

Vortex shedding in the wake of bluff bodies is an important flow phenomenon. At subsonic and transonic speeds, it has long been recognized that the wake behind an isolated two-dimensional section with a blunt trailing edge may break into a vortex street. The direct result of this is an increase in drag, mainly as a result of reduced pressure. Further, the subject of base flows at high Reynolds numbers has been and continues to be an important area of research in view of its relevance in external aerodynamics. Base drag arising from flow separation at the blunt base of a body, can be sizeable fraction of total drag in the context of projectiles, missiles and after bodies of fighter aircraft; for example, the base drag component can be as high as 50 percent of the total drag for a missile with power off (i.e. with no jet flow at the base). Large-scale flow unsteadiness, often associated with a turbulent separated flow, can cause additional problems like base buffeting which are undesirable. Because of its vide applicability, suddenly expanded flows have studied extensively. Many researchers attempted to control the base pressure with passive means and some of the works relevant to the present study are reviewed in the section to follow. Therefore, in the present study an attempt is made to investigate the base pressure control with active control in the form of micro jets.

\section{LITERATURE REVIEW}

Hall and Orme [1] studied compressible flow through sudden enlargement in a pipe, both theoretically and experimentally, and showed a good agreement between theoretical and experimental results. They developed a theory to predict the Mach number in a downstream location of sudden enlargement for known values and Mach number at the exit of the inlet tube, with incompressible flow assumption. They also assumed that the pressure across the face of the enlargement was equal to the static pressure in the small tube just before the enlargement. But this assumption is far away from reality, it is a well established fact that the pressure across the face in the recirculation region, namely the base pressure is very much different from the pressure in the smaller tube just before the enlargement. They used a nozzle and tube arrangement for the experiments and studied the problem with a range of throat Mach numbers from 0.0 to 1.0. Korst [2] wrote comment on the boundary layer effects on sonic flow through an abrupt cross-sectional area change. He compared his theoretical results which utilize a two-dimensional flow model considering the interaction between dissipative flow regions and the adjacent free stream with Wicks results and showed good agreement between theory and experiments. Benedict [3] with various other investigators analyzed the sudden enlargement problem in an elaborate manner both theoretically and experimentally. Drewry [4] performed experimental studies to axisymmetric, sudden expansion dump combustor configurations for integral rocket-ramjet missile under cold flow 
test conditions. These studies have included surface flow visualization, measurements of total and static pressures, and gas sampling within a representative combustor duct. Flow visualizations tests, using a surface oil flow technique, vividly demonstrated the complex nature of the flow recirculation region downstream of the dump station. Gas concentration measurements of simulated fuel-air mixing were made in the combustor duct using an on-line gas analysis system with a quadruple mass spectrometer. Combustor flow field studies were made for test configurations with and without mechanical flame holding devices. Direct correlations between cold flow mixing data and measurements of combustion efficiency from direct-connect ram burner tests were made as a function of combustor length-to-diameter ratio. Eaton and Johnston [5] wrote a review paper on research of subsonic turbulent reattachment. They described the general features of backward-facing step flow. Five principal independent parameters on reattachment length, 1) initial boundary-layer state, 2) initial boundary-layer thickness, 3) free stream turbulence, 4) pressure gradient, and 5) aspect ratio were discussed in the review Berbee and Ellzey [6] studied the effect of aspect ratio on the flow over a rear-ward-facing step. From their experimental results they concluded that the mean velocity and turbulence intensity profiles are constant across the width of the test-section for either of the Reynolds numbers tested for aspect ratios of 10 and 4. At a distance greater than three step height downstream of the step, the peak turbulence intensity is greater for a higher aspect ratio and is relatively insensitive to Reynolds number. The peak frequency is lower and the spectrum is narrower for a higher aspect ratio in the region near the step. Flow through a rectangular Passage which is expanded suddenly into another rectangular duct of larger Cross-sectional area has been studied experimentally with stagnation Pressures from 3.5 atmospheres to 1.25 atmospheres by Rathakrishnan et al. [7]. The length to height ratio of the enlarged duct varied from 5.769 to 1.923 and three models with length to height ratios 5.769, 3.846, and 1.923 were studied. The influence of stagnation Pressures and length to height ratio of the enlarged duct on base pressure and flow field mean pressures in the enlarged duct is discussed. The results of the present investigation indicate that the oscillatory nature of the mean pressure flow field in the enlarged portion with rectangular cross-section is appreciably different from that for circular cross-section at similar flow conditions. Shaw et al. [8] did conditional analysis of wall pressure fluctuations in plume-induced separated flow fields. The separation process in plume-induced, boundary-layer separated flow fields was found to be unsteady. Khan and Rathakrishnan [9-13] done experimental investigation to study the effectiveness of micro jets under the influence of Over, Under, and Correct expansion to control the base pressure in suddenly expanded axi-symmetric ducts. They found that the maximum increase in base pressure is 152 percent for Mach number 2.58. Also they found that the micro jets do not adversely influence the wall pressure distribution. They showed that micro jets can serve as an effective controller raising the base suction to almost zero level for some combination for parameters. Further, it was concluded that the nozzle pressure ratio has a definite role to play in fixing the base pressure with and without control. The effectiveness of micro jets to control the base pressure in suddenly expanded axi-symmetric ducts is studied experimentally by Ashfaq et al. [14-17]. From the experimental results, it was found that the micro jets can serve as active controllers for base pressure. From the wall pressure distribution in the duct it found that the micro jets do not disturb the flow field in the enlarged duct. They presented the results of experimental studies to control the base pressure from a convergent nozzle under the influence of favourable pressures gradient at sonic Mach number. The area ratio (ratio of area of suddenly expanded duct to nozzle exit area) studied are 2.56, 3.24, 4.84 and 6.25. The L/D ratio of the sudden expansion duct varies from 10 to 1 . They concluded that, unlike passive controls the favourable pressure gradient does not ensure augmentation of the control effectiveness for active control in the form of micro jets. Wall pressure was measured and it is found that the micro jets do not disturb the flow field in the duct rather the quality of flow has improved due to the presence of micro jets in some cases.

In the present study our focus is to understand the flow field in the enlarged duct when the control in the form of micro jets are activated; and how the control is going to influence the flow field. Since, one of the common problems encountered in suddenly expanded flow field is that the pressure field in the enlarged duct becomes oscillatory whenever; passive or active controls are employed. These oscillations are reflected as variation in the wall pressure distribution of the enlarged duct. Therefore, it becomes mandatory on the part of a researcher working on sudden expansion problems to monitor wall pressure distributions in the enlarged duct. In other words when we employ a control to modify the base pressure level, there is a possibility that the control might augment the oscillatory nature of the flow field in the enlarged duct. To account for this undesirable effect (aggravating the oscillatory nature of the flow field) wall pressure distribution in the enlarged duct was measured for all combination of parameters of the present investigation.

\section{EXPERIMental Procedure}

Fig. 1 shows the experimental setup used for the present study. At the exit periphery of the nozzle there are eight holes as shown in figure 1, four of which are (marked c) were used for blowing and the remaining four (marked $\mathrm{m}$ ) were used for base pressure $\left(\mathrm{P}_{\mathrm{b}}\right)$ measurement. Control of base pressure was achieved by blowing through the control holes (c), using pressure from a settling chamber by employing a tube connecting the main 
settling chamber with the control chamber, and, the control holes (c). Wall pressure taps were provided on the duct to measure wall pressure distribution. First nine holes were made at an interval of $3 \mathrm{~mm}$ each and remaining was made at an interval $5 \mathrm{~mm}$ each. From literature it is found that, the typical L/D (as shown in Fig. 1) resulting in $P_{b}$ maximum is usually from 3 to 5 without controls. Since active controls are used in the present study, L/D ratios up to 10 have been employed.

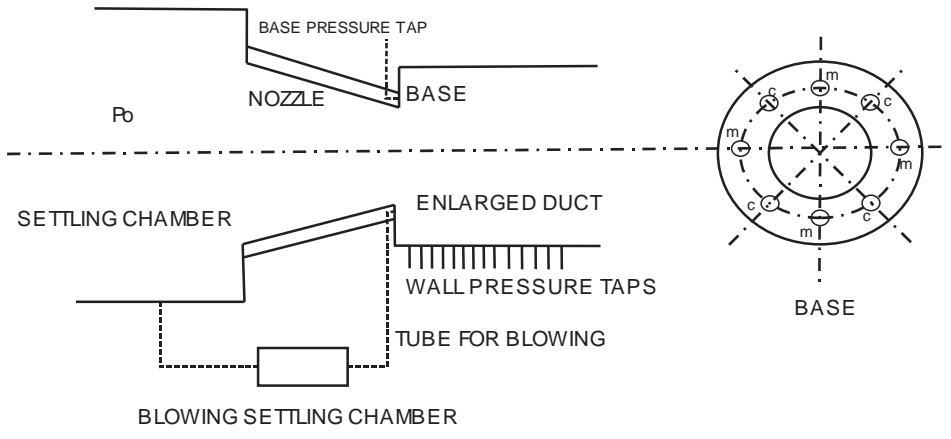

Figure 1: Experimental Setup

The experimental setup of the present study consisted of an axi-symmetric nozzle followed by a concentric axi-symmetric duct of larger cross-sectional area. The exit diameter of the nozzle was kept constant (i.e. $10 \mathrm{~mm}$ ) and the area ratio of the model was 2.56 defined, as the ratio of the cross-sectional area of the enlarged duct to that of the nozzle exit, was achieved by changing the diameter of the enlarged duct. The suddenly expanded ducts were fabricated out of brass pipe. Model length was ten times the inlet diameter so that the duct has a maximum $L / D=10$. The lower $\mathrm{L} / \mathrm{Ds}$ were achieved by cutting the length after testing a particular L/D.

PSI model 9010 pressure transducer was used for measuring pressure at the base and the stagnation pressure in the settling chamber. It has 16 channels and pressure range is 0-300 psi. It averages 250 samples per second and displays the reading. The software provided by the manufacturer was used to interface the transducer with the computer. The user-friendly menu driven software acquires data and shows the pressure readings from all the 16 channels simultaneously in a window type display on the computer screen. The software can be used to choose the units of pressure from a list of available units, perform a re-zero/full calibration, etc. The transducer also has a facility to choose the number of samples to be averaged, by means of dipswitch settings. It could be operated in temperatures ranging from $-20^{\circ}$ to $+60^{\circ} \mathrm{C}$ and 95 per cent humidity.

\section{RESULTS AND DISCUSSION}

The measured data consists of base pressure $\left(\mathrm{P}_{\mathrm{b}}\right)$; wall static pressure $\left(\mathrm{P}_{\mathrm{w}}\right)$ along the duct and the nozzle pressure ratio (NPR) defined as the ratio of stagnation pressure $\left(\mathrm{P}_{0}\right)$ to the back pressure $\left(\mathrm{P}_{\mathrm{atm}}\right)$. All the measured pressures will be non-dimensionalized by dividing them with the ambient pressure (i.e. the back pressure). In the present study the pressure in the control chamber will be the same as the NPR of the respective runs since we have drawn the air from the main settling chamber. One of the common problems encountered in suddenly expanded flow field is that the pressure field in the enlarged duct becomes oscillatory whenever; passive or active controls are employed. To quantify the effect of control on wall pressure distribution $\mathrm{P}_{w} / \mathrm{P}_{\mathrm{a}}$ for the two cases, namely with and without control have been compared.

Figs. 2(a) to (f) and Figs. 3(a) to (b) present the wall pressure distribution in the enlarged duct for area ratio 2.56, for L/D 10 to 1 for correctly expanded case at Mach number $M=0.9$. From the figure it is seen that there is some influence on the wall pressure field in the base region and the wall pressure values for with and without control cases remains the same and this oscillatory nature starts in the vicinity of the base region extending up to $\mathrm{x} / \mathrm{L}=0.35$. Since, the flow is correctly expanded; therefore, the shear layer which is expanding freely from the nozzle is strongly influenced by the additional relief available to the flow, which; in turn forms expansion waves downstream after exiting from the nozzle. Therefore, flow coming out of the nozzle will have a tendency to deflect away from the shock, under such circumstances, when the micro jets are activated the entrainment of the micro jets is bound to carry some mass from the base region. It is also, seen that the magnitude of the wall pressure is fluctuating due the presence of the waves in the flow region. Hence, the assumption in the scientific community that the correctly expanded flows will be free from the waves is again proved wrong; in Figs. 2(a) and (b) for L/D ratio = 10 and 8, it has been clearly demonstrated. It is also seen that once the flow has crossed the reattachment point the flow in the downstream becomes smooth.

Figs. 2(c) to (d) present the wall pressure results for $\mathrm{L} / \mathrm{D}=6$ and 5 . The wall pressure behavior in these figures is different from the previous figures. From $\mathrm{x} / \mathrm{L}=0.0$ to 0.2 the wall pressure assumes constant value; 
then there is a jump in the duct wall pressure value at the non-dimensional location $\mathrm{x} / \mathrm{L}=0.4$, further, in the downstream there is a smooth increase in the wall pressure, it is also seen that the flow field with and without control remains the same for both the cases. Results for $\mathrm{L} / \mathrm{D}=6$ and 5 once again proved that the correctly expanded flows are wave dominated, the reason for this trend could be that we are considering the flow Mach number $\mathrm{M}=0.9$, when the flow is coming out from the nozzle experiencing sudden increase in the area which will result in further expansion of the flow, the flow will undergo compression, expansion, reflection from the duct wall, and the recombination; all these activities are taking place within the reattachment length.

Fig. 2(e) presents wall pressure results for $\mathrm{L} / \mathrm{D}=4$. It is seen that the wall pressure values are constant till $\mathrm{x} / \mathrm{L}=0.3$, there is small kink and then further downstream flow becomes smooth and pressure recovery is linear. The reasons for this non-oscillatory behavior of the wall pressure field may be due to the short duct length, the influence of the back pressure to the flow field. The wall pressure results for $\mathrm{L} / \mathrm{D}=3$ are shown in Fig. 2(f), from the figure it is seen that wall pressure values for the initial three pressure taps has marginally increased, then further, downstream there are small fluctuations in the wall pressure for about four pressure taps then wall pressure recovery is smooth. Also, it is seen that the wall pressure value at $\mathrm{x} / \mathrm{L}=0$ which was around 0.62 has increased to a level around 0.75 and this high value is mainly due to the influence of the back pressure, the oscillations and sudden jumps in the wall pressure which were seen for the higher L/Ds are absent, and smooth increase in the wall pressure is seen.

Similar results are seen in Fig. 3(a) for $\mathrm{L} / \mathrm{D}=2$, the only difference in the results for $\mathrm{L} / \mathrm{D}=2$ with that of for $\mathrm{L} / \mathrm{D}=3$, is that the initial value of the wall pressure has slightly increased, here also it seen that the wall pressure values for first three pressure taps are constant which indicates that this happens to be within the reattachment length and in the downstream flow development is very smooth. For $\mathrm{L} / \mathrm{D}=1$, the results are shown in Fig. 3(b) these results clearly indicate that this length of the duct is not sufficient for the flow to be attached with the duct wall hence, the flow is behaving as a free jet.

Results for Mach number $M=0.8$ are presented in Figs. 3(c) to (f) and Figs. 4(a) to (d). Results presented by Figs. 3(c) to (d) are on the similar lines as we have seen for Mach number M $=0.9$ for L/D 10 and 8. The only difference in the present case and the previous case, that there is decrease in the Mach number from 0.9 to 0.8 , which means that there is a slight reduction in the inertia value. In view of the decrease in the inertia, the initial value of wall pressure has marginally increased. For the initial eleven pressure taps which happens to be within the reattachment length the flow field remains oscillatory and the reasons for this nature are same as discussed above for Mach 0.9. In the downstream the flow field is smooth. Figs. 3(e) to (f) presents results for $\mathrm{L} / \mathrm{D}=6$ and 5. It is seen that the trend is almost identical to that of Fig. 2(c) and (d) with the exception that the magnitude of wall pressure is marginally increased as compared to that of for Mach number $\mathrm{M}=0.9$. Here, also it is seen that the value of the wall pressure for initial three taps is constant then there is a smooth increase; further downstream there is small jump in the wall pressure and later the flow development is smooth, the values for with and without control are same which implies that the flow field is undisturbed.

Figs. 4(a) to (b) show the results for $\mathrm{L} / \mathrm{D}=4$ and 3, here again we see the similar results as that of for Mach number $\mathrm{M}=0.9$, with initial increase in the value of the wall pressure since for the initial taps which are within the base region which is influence by the base vortex and further downstream the flow becomes smooth. For this particular L/D the major contributor to influence the flow are the influence of atmospheric pressure, as well as the entrainment of the flow from the surrounding area, and due to the turbulent mixing effect. For L/D = 2 and 1, the results are shown in (Fig. 4(c) to (d)). From the figure it is seen that for L/D $=2$ (Fig. 4(c)) the wall pressure assumes very high value for the initial three taps and later the flow attains almost the value of atmospheric pressure. Results for L/D = 1 are shown in Fig. 4(d) from the figure it is clear that the flow is no more attached with the duct wall and once again it behaves as free jets without getting influenced by the base region as well as the micro jets.

The wall pressure distribution for Mach number $M=0.6$ are presented in Figs. 4(e) to (f) and Figs. 5 (a) to (f) for $\mathrm{L} / \mathrm{D}=10,8,6,5,4,3,2$ and 1, respectively. Figs. 4(e) to (f) present the results for $\mathrm{L} / \mathrm{D}=10 \& 8$. If we compare these results with those for earlier two Mach number $M=0.9$ and 0.8 , it is found that due to the decrease in the Mach number, the wall pressure has achieved a high value and as high as 30 percent increase in the initial value, also, it is seen that the oscillations in the base region are there but there is definite reduction in the magnitude of the wall pressure and this happens only for initial three taps later oscillations are suppressed significantly, later beyond the reattachment length the flow development is smooth. The reasons for this behavior may due to decrease in the Mach number and inertia available to the flow has reduced significantly and for this area ratio the relief enjoyed by the suddenly expanded flow is the same. Hence, for the same area ratio the level of expansion causing the formation of shock wave or expansion fan at the nozzle lip will have a strong effect on the wall pressure as well as its control effectiveness. These results imply that the flow field becomes sensitive to the level of expansion, inertia available at the nozzle exit and the relief available at the expanded plane. 


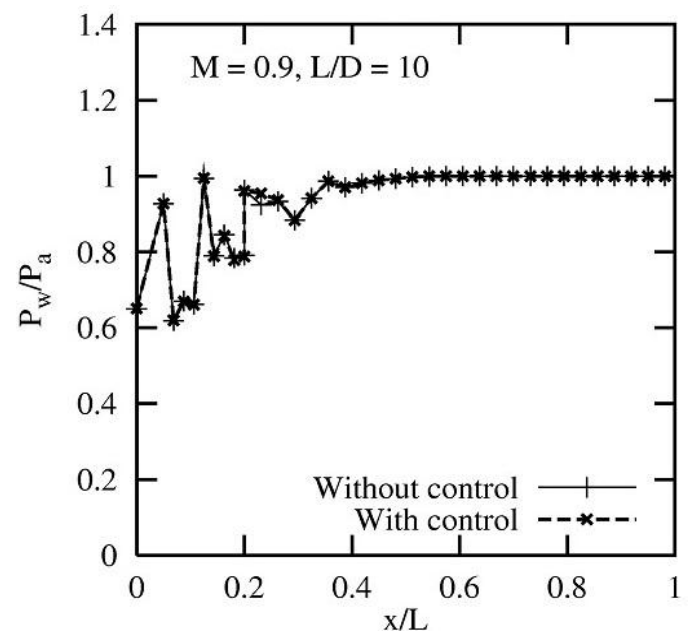

(a)

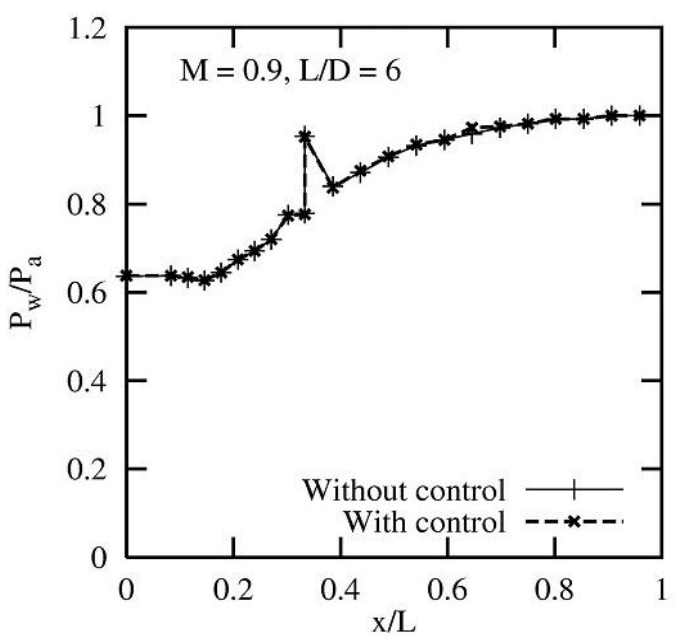

(c)

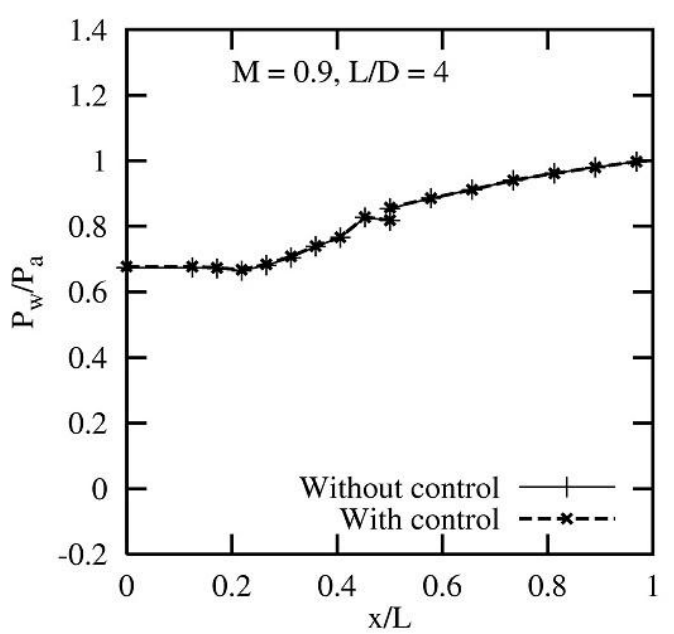

(e)

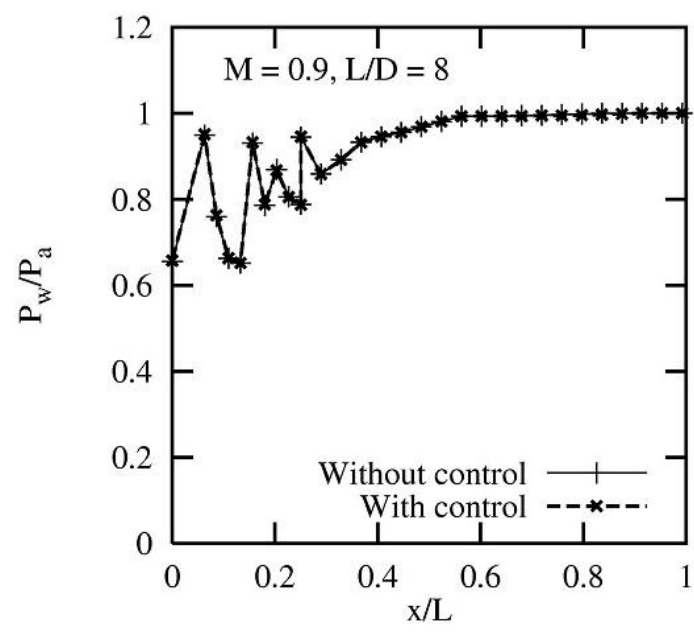

(b)

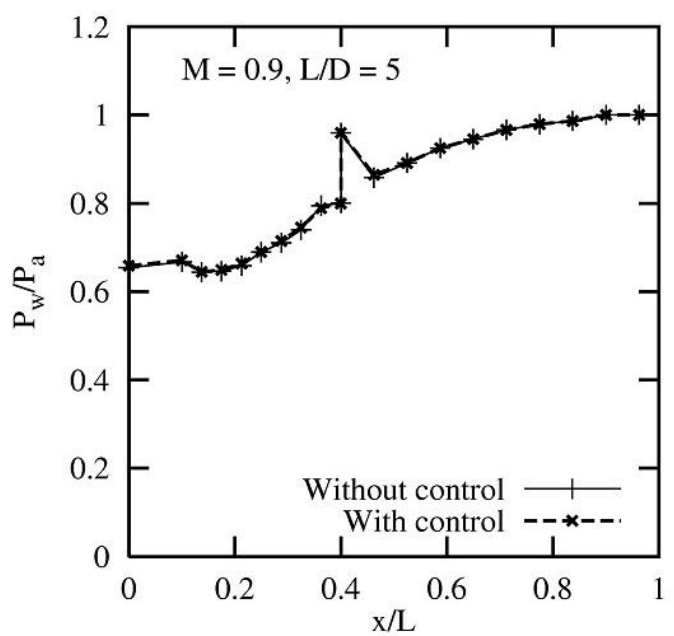

(d)

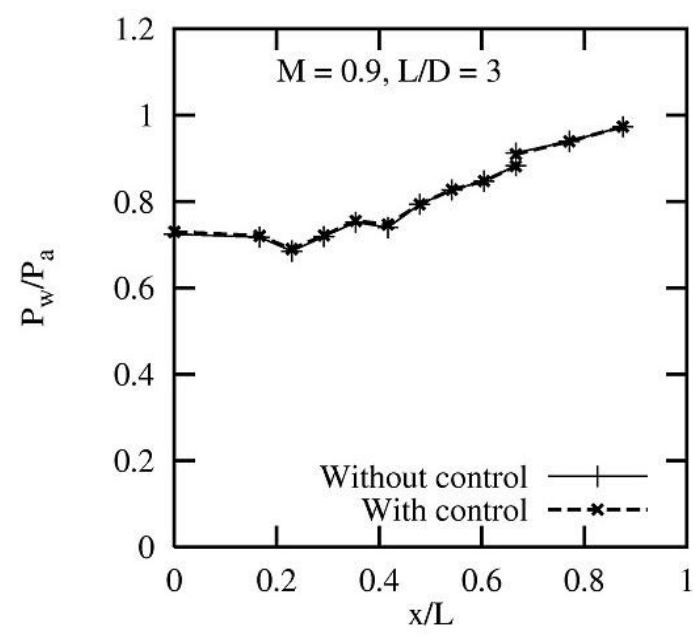

(f)

Figure 2: Wall pressure distribution 


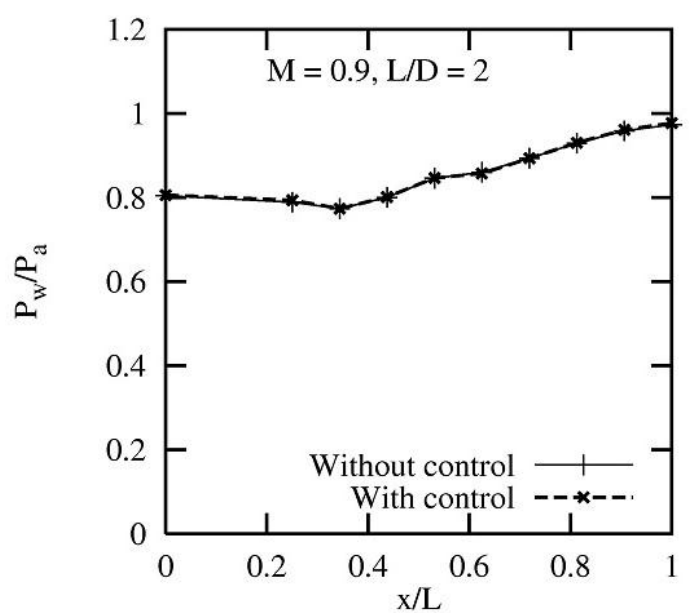

(a)

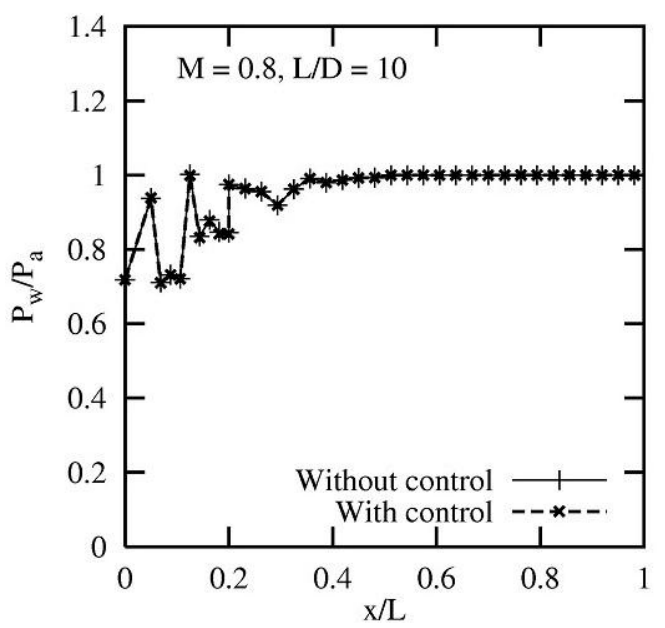

(c)

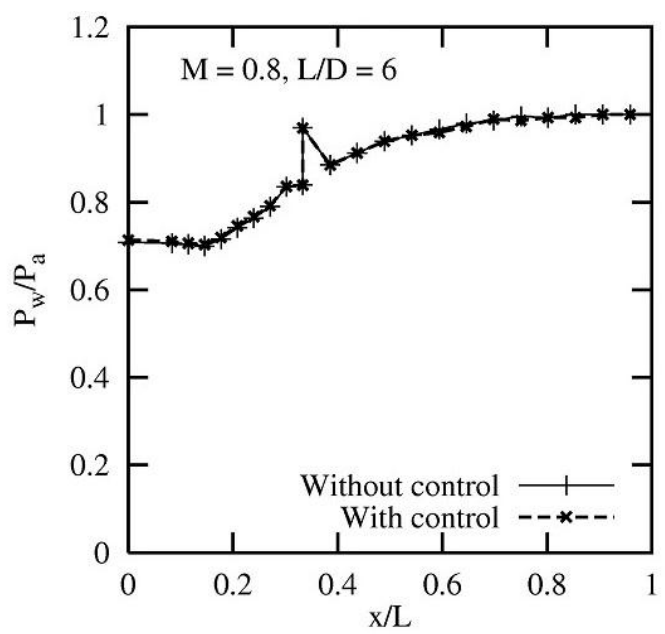

(e)

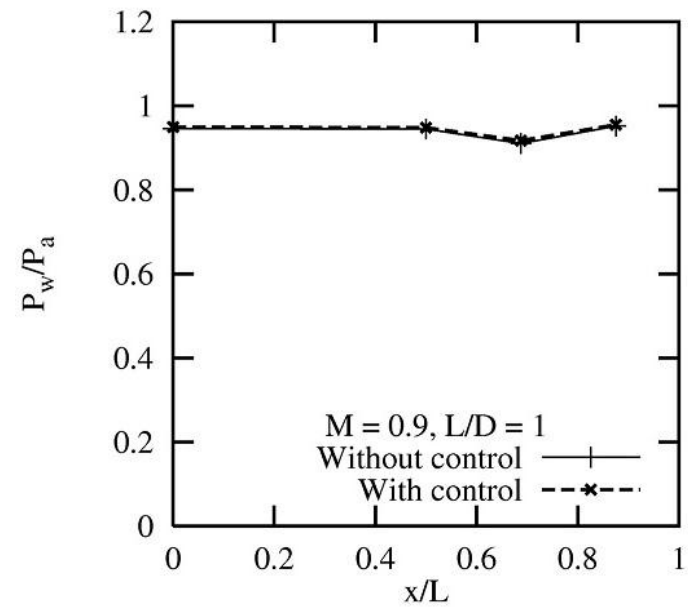

(b)

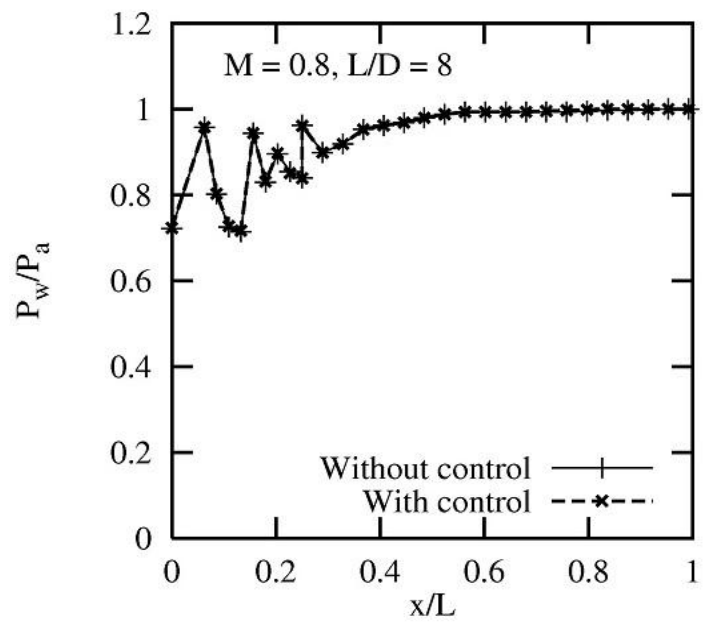

(d)

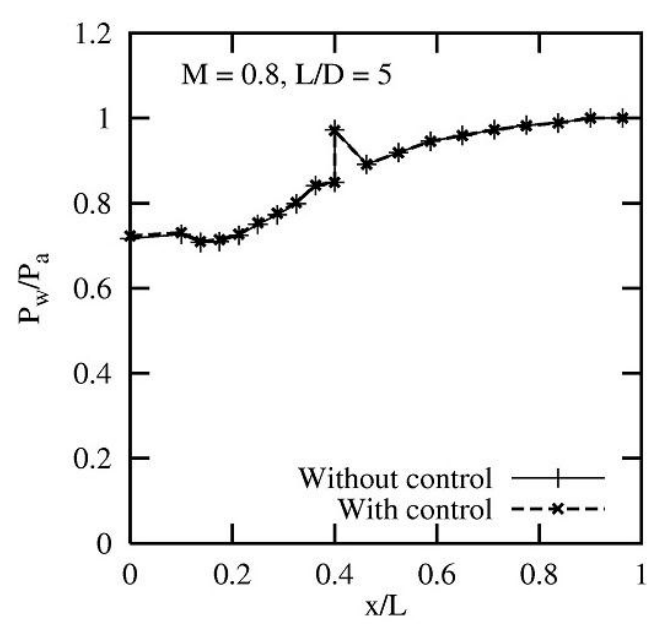

(f)

Figure 3: Wall pressure distribution 


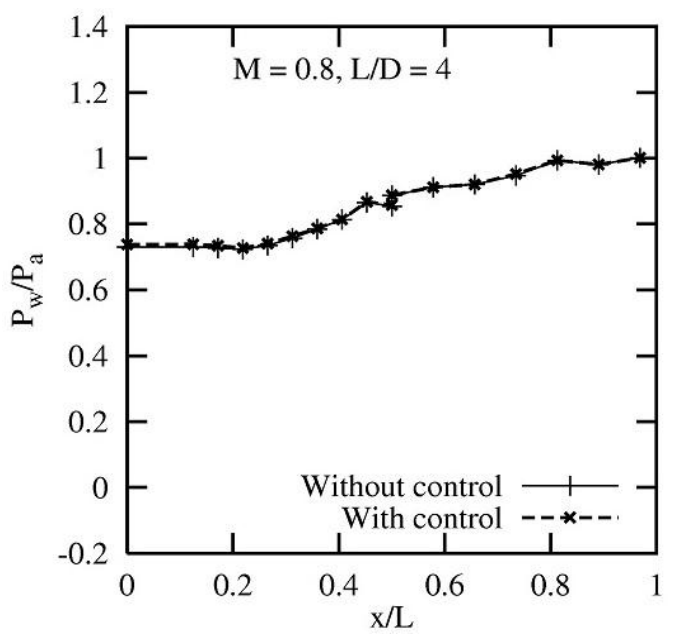

(a)

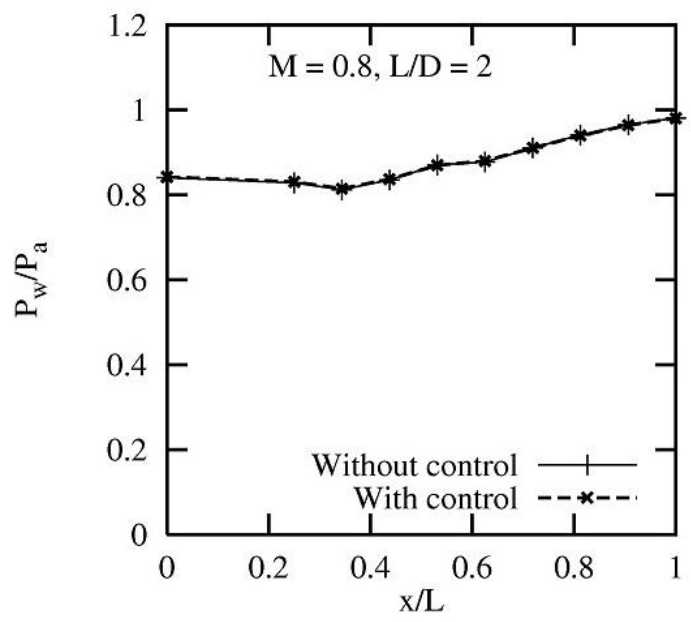

(c)

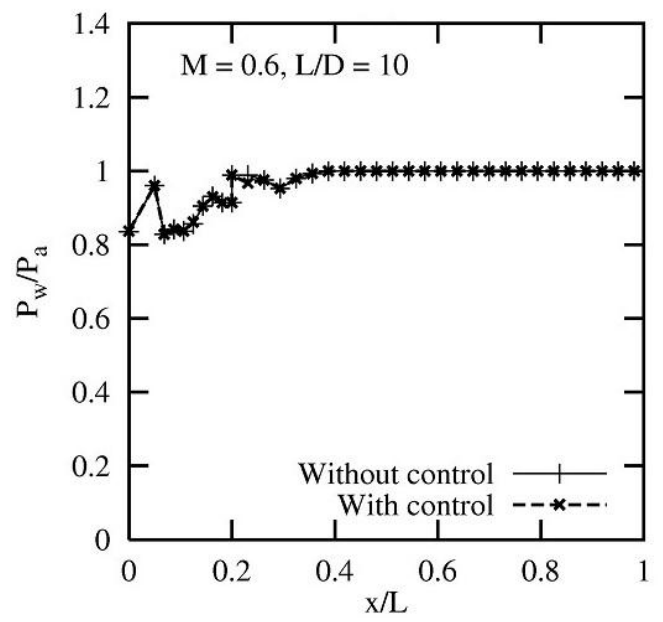

(e)

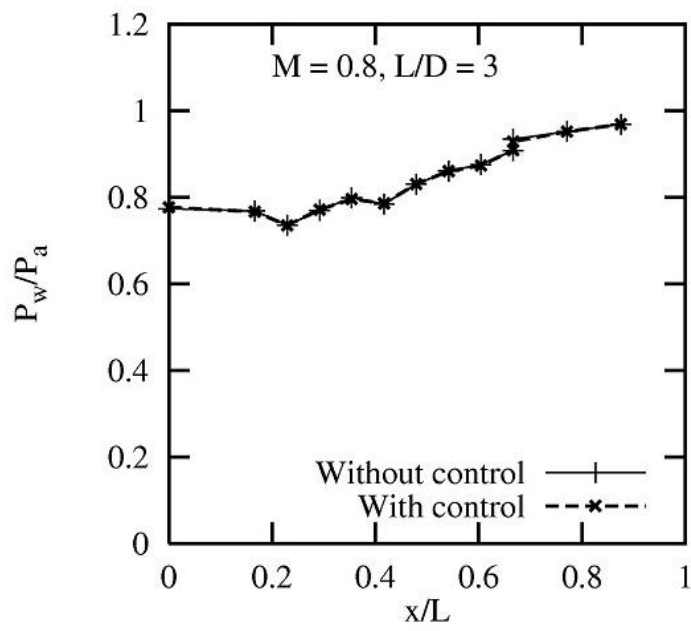

(b)

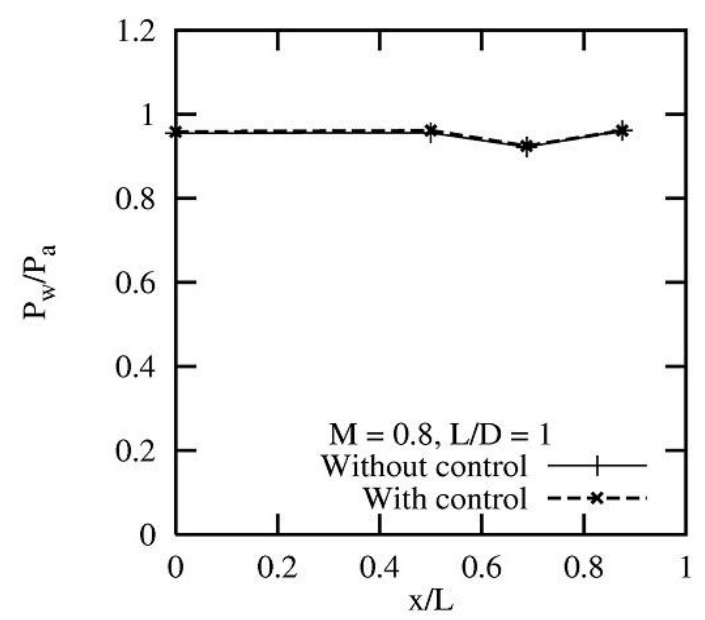

(d)

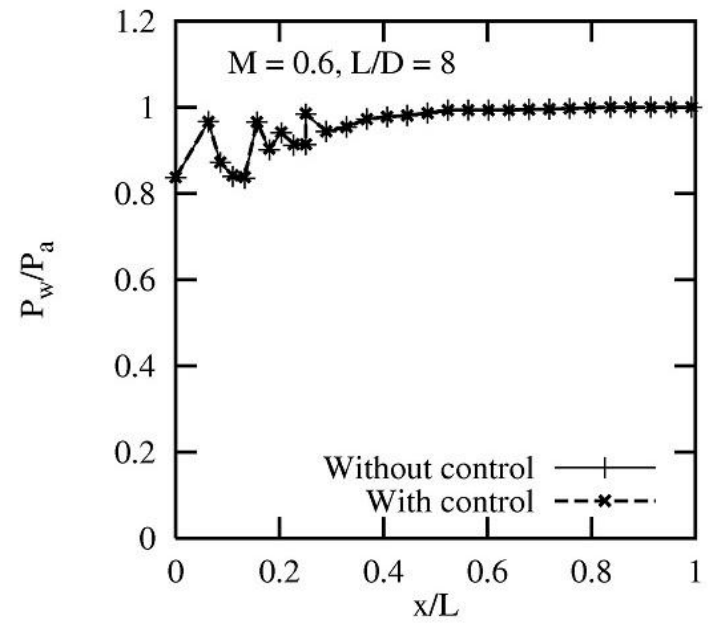

(f)

Figure 4: Wall pressure distribution 


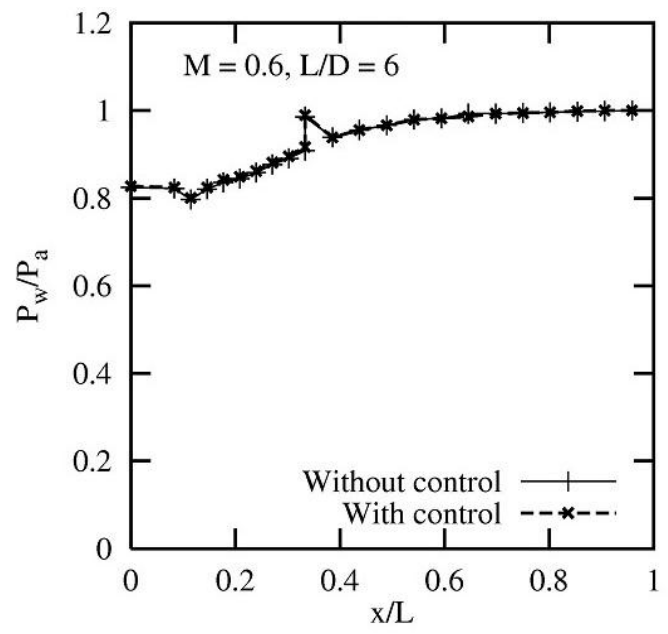

(a)

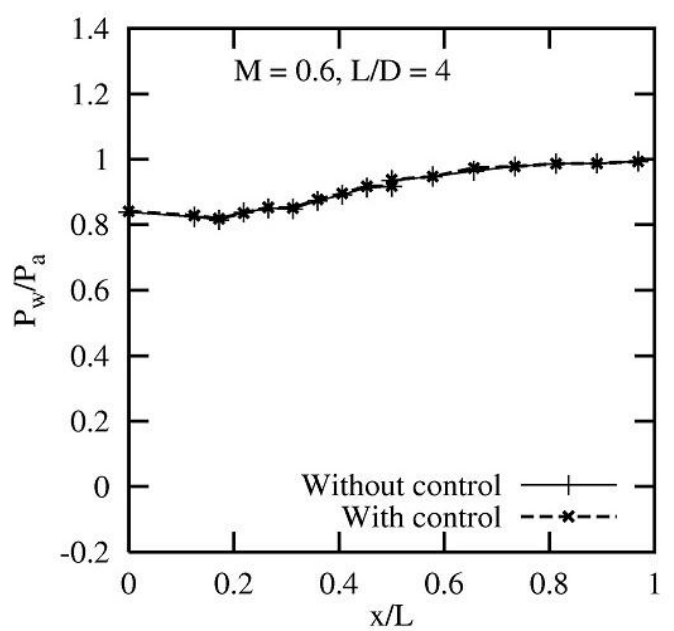

(c)

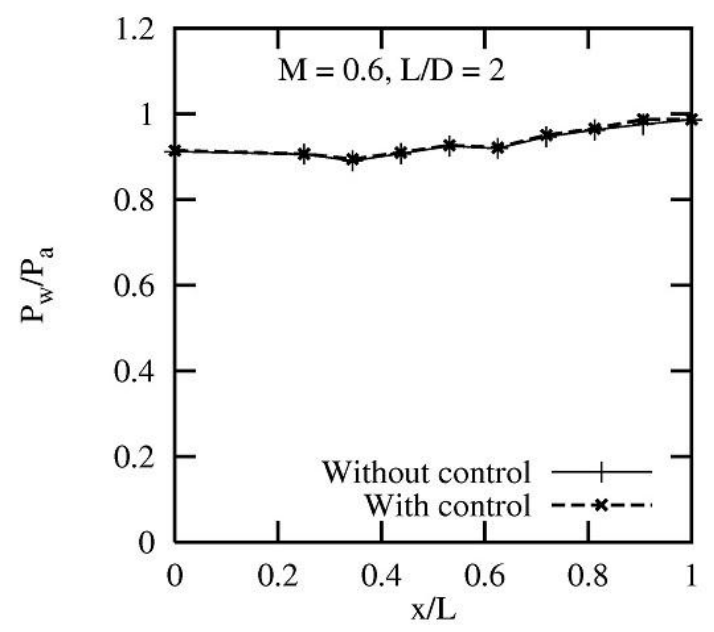

(e)

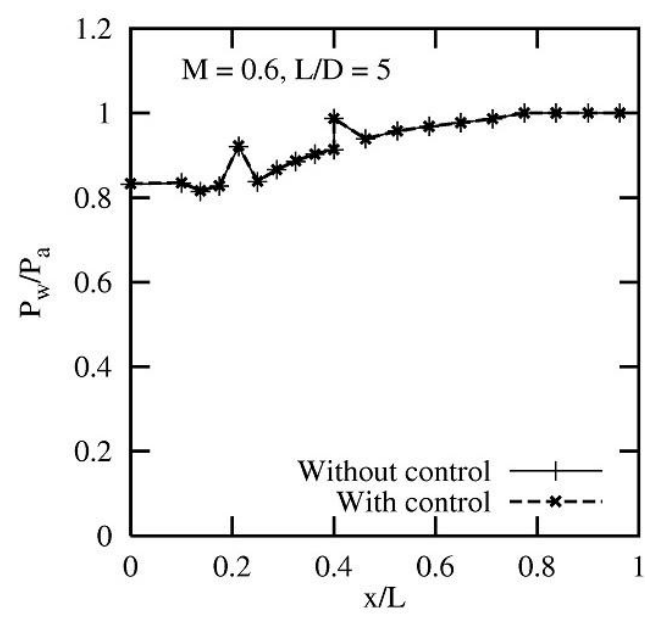

(b)

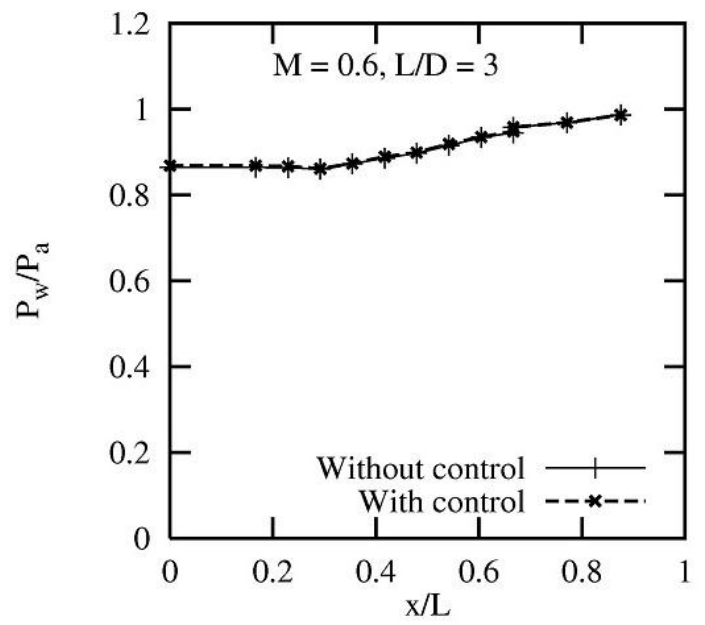

(d)

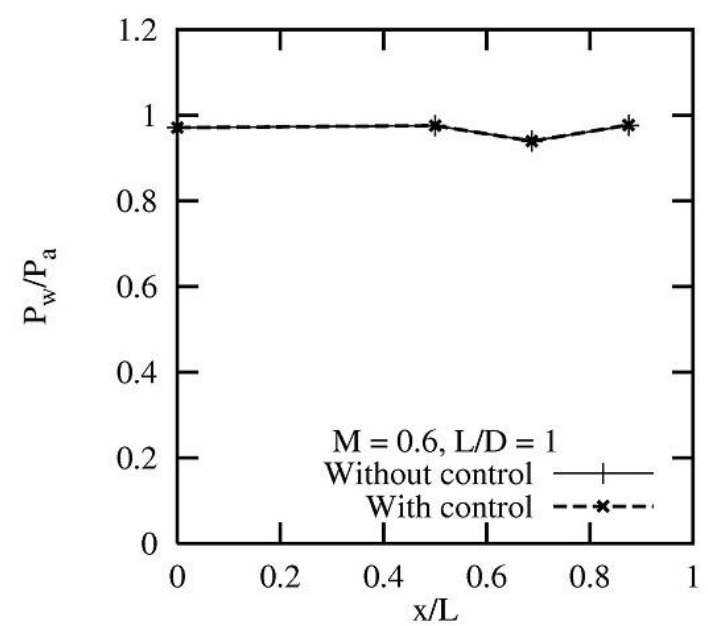

(f)

Figure 5: Wall pressure distribution 
Fig. 5(a) to (b) presents the wall pressure results for $\mathrm{L} / \mathrm{D}$ ratios $=6$ and 5. Here, again there is about 20 percent increase in initial value of the wall pressure as compared to Mach number $M=0.8$. From the figure it is observed that a shock is positioned at $\mathrm{x} / \mathrm{L}=0.35$ due to which the wall pressure has increased by 20 percent and then for the next wall pressure tap it has come down to the same value as it was for the previous pressure tap. In Fig. 5(b) it is seen that there is sudden jump in the wall pressure value at two locations in the wall duct, first at $\mathrm{x} / \mathrm{L}=0.2$ and next at $\mathrm{x} / \mathrm{L}=0.4$, then further downstream the flow development is very smooth. It may be due to the interaction of the shock wave, reflection of the shock from the wall, interaction of the free shear layer, and interaction with the vortex in the base region. This peculiar phenomenon reiterates which we have stated initially that correctly expanded flow is not free from the wave but it is wave dominated.

Figs. 5(c) to (d) presents the results for $\mathrm{L} / \mathrm{D}=4$ and 3, from the figure it is seen that there is increase in the initial value of the wall pressure and the flow is non-oscillatory as compared to the results for same L/D for higher Mach numbers. The wall pressure has increase by 25 to 35 percent for the same condition for Mach numbers $\mathrm{M}=0.8$ and 0.9 .

Fig. 5(d) presents the results for $\mathrm{L} / \mathrm{D}=3$, the trend is same as that of for higher Mach numbers and there is a increase in the value of wall pressure as compared to the previous cases for the same L/D ratio. The wall pressure has gone up by 40 percent as compared to that for Mach number $\mathrm{M}=0.9$ for the same L/D ranges. Figs. 5(e) to (f) presents the wall pressure results for $\mathrm{L} / \mathrm{D}=2$ and 1 . From the figure it is clearly visible that the flow is no more attached with the duct wall for these cases, the jet behaves as free jet.

\section{CONCLUSION}

From the above results we can draw the following conclusions:

- The flow field in the wall duct is dominated by the presence of waves, reflection from the wall, recompression and recombination's.

- The flow from the nozzle with correct expansion is not free from the waves as it can be seen from the wall pressure flow field.

- The flow field is oscillatory within the base region and beyond the reattachment points the development of the flow and the wall pressure recovery is very smooth. This happens for $\mathrm{L} / \mathrm{D}=10,8,6$, and 5 only.

- With decrease in the Mach number which results in decrease in inertia also results in increase of wall pressure progressively.

- The minimum duct length requirement seems to be $\mathrm{L} / \mathrm{D}=2$ for the parameters of the present study.

\section{REFERENCES}

[1] W. B. Hall and E. M. Orme, Flow of a compressible fluid through a sudden enlargement in a pipe, Proceedings of Mechanical Engineers, Vol. 169, 1956, pp. 1007-1022.

[2] H. Korst, Comments on the effects of boundary layer on sonic flow through an abrupt cross sectional area change, Journal of the Aeronautical Science, Vol. 21, 1954, pp. 568-569.

[3] R. P. Benedict and N. A. Carlucci, Flow with losses, Trans ASME (Power) Vol. 87, 1965, pp. 37-49.

[4] J. E. Drewry, Fluid dynamic characteristics of sudden expansion ram jet combustors flow fields, AIAA Journal, Vol. 16, No. 4, 1978, pp. 313-319.

[5] J. K. Eaton and J. P. Johnston, A review of research on sub sonic turbulent flow re attachment, AIAA journal, Vol. 119, No.9, 1981, pp. $1093-1100$.

[6] J. G. Berbee and J. L. Ellzey, The effect of aspect ratio on the flow of over a rear-ward-facing step, Experiments in Fluids, 7, 1989, pp. 447-452.

[7] E. Rathakrishnan, T. J. Ignatius and Channa Raju, An experimental study of Suddenly Expanded Flow field, Mechanics Research Communications, Vol. 18(1), 1991 pp. 1-9.

[8] R. J. Shaw, J. C. Dutton, and A. L. Addy, Conditional analysis of wall pressure fluctuations in plume-induced separated flow fields, AIAA Journal, Vol. 37, No. 11, November 1999, pp. 1436-1443.

[9] S. A. Khan and E. Rathakrishnan, Active Control of Suddenly Expanded Flows from Over expanded Nozzles, International Journal of Turbo and Jet Engines (IJT), Vol. 19, No. 1-2, 2002, pp. 119-126.

[10] S. A. Khan and E. Rathakrishnan, Control of Suddenly Expanded Flows with Micro Jets, International Journal of Turbo and Jet Engines (IJT), Vol. 20, No.2, 2003, pp. 63-81.

[11] S. A. Khan and E. Rathakrishnan, Active Control of Suddenly Expanded Flow from Under Expanded Nozzles, International Journal of Turbo and Jet Engines, (IJT), Vol. 21, No. 4, 2004, pp. 233-253.

[12] S. A. Khan and E. Rathakrishnan, Control of Suddenly Expanded Flow from Correctly Expanded Nozzles, International Journal of Turbo and Jet Engines (IJT), Vol. 21, No. 4, 2004, pp. 255-278.

[13] S. A. Khan and E. Rathakrishnan, Control of Suddenly Expanded Flow, Aircraft Engineering and Aerospace Technology: An International Journal, Vol. 78, No. 4, 2006, pp. 293-309.

[14] Syed Ashfaq, S. A. Khan and E. Rathakrishnan, Active Control of Flow through the Nozzles at Sonic Mach Number, International Journal of Emerging Trends in Engineering and Development, Vol. 2, Issue-3, 2013, pp. 73-82.

[15] Syed Ashfaq and S. A. Khan, Sonic Under Expanded Flow Control with Micro Jets, International Journal of Engineering Research and Applications, Vol. 3, Issue-6, 2013, pp. 1482-1488.

[16] Syed Ashfaq, S. A. Khan and E. Rathakrishnan, Control of suddenly expanded flow for area ratio 3.61, International Journal of Advanced Scientific and Technical Research, Issue 3 volume 6, Nov.-Dec. 2013, pp. 798-807.

[17] Syed Ashfaq and S. A. Khan, Experimental Studies on Low Speed Converging Nozzle Flow with Sudden Expansion, International Journal of Emerging Technology and Advanced Engineering, Volume 4, Issue 1, January 2014, pp. 532-540. 